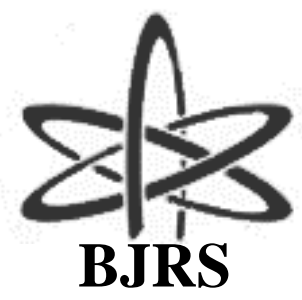

\author{
BRAZILIAN JOURNAL \\ $\mathrm{OF}$ \\ RADIATION SCIENCES \\ 09-01A (2021) 01-11
}



\title{
A resorbable scaffold for bone replacement
}

\author{
Anessi $^{\mathrm{a}}$ M.C., Bustamante ${ }^{\mathrm{a}}$ P.A., Horak ${ }^{\mathrm{a}}$ C.I., Villares ${ }^{\mathrm{b}}$ B. \\ ${ }^{a}$ Ezeiza Atomic Centre/Radiation Processes Department/Biological Application Division, B1802AYA, Ezeiza, Buenos \\ Aires, Argentina \\ canessi@cnea.gob.ar \\ pbustamante@cnea.gob.ar \\ celina.horak@gmail.com \\ ${ }^{b}$ Argentine Nanotechnology Foundation/1650, San Martín, Buenos Aires, Argentina
}

\begin{abstract}
All tissue banking activities are depending on tissue donors. The donor rate is still low in Argentina, and the tissue demanding is still not fulfilled. For this reason, tissue engineering has become a necessary discipline to be investigated. Our project is conducted to obtain a 3D resorbable printed scaffold seeded with mesenchymal stem cell (MSC), to conduct real bone. We produced three polylactic acid (PLA) filaments with different loads of hydroxyapatite (HA): $3 \%, 5 \%$ and $10 \%$. The mixtures were homogenous and the three filaments were suitable for 3D printing and were used to print 3D scaffolds samples. The scaffolds were irradiated with range doses of 15 kGy to $25 \mathrm{kGy}$ for sterilization purposes and to evaluate if the degradation polymer rate is regulated with the irradiation dose. The elaborated filaments were optimally printable. In addition they turned out to be not cytotoxic (cell viability greater than $\mathbf{7 0 \%}$ ) and whit good cellular adherence. In this way, our biomaterial seems to be good for scaffolds for bone replacement.
\end{abstract}

Keywords: scaffold, 3D printing, resorbable, biomaterial, radiation. 


\section{INTRODUCTION}

Tissue engineering is applied to regeneration bone and is a multidisciplinary area in which biological, chemical, physical and engineering principles are applied for the development of these [1,2]. To fulfill this objective, osteoprogenitor cells are required for their multiplication and production of extracellular matrix and scaffolds made of biomaterials as a support structure that guides cell growth, facilitating adhesion, migration, proliferation and cellular stability $[1,2]$ In the case of materials for bone replacement, the idea is to create a three-dimensional material capable of supporting the initial loads and that is progressively degraded so the material is intended to be reabsorbed once the tissue re-establishes its function [3, 4].

The most commonly used materials for the development of three-dimensional structures are hydroxyapatites (HA), biodegradable polymers and some natural polymers [3]. The HA is a ceramic material that constitutes the inorganic phase of bone to facilitate osteointegration and osteoconduction. Polylactic acid (PLA) is a synthetic absorbable polymer of the family of alpha hydroxy acids or aliphatic polyesters, which is biocompatible and biodegradable in an immunologically inert, non-toxic and resorbable compound [3].

For a three-dimensional scaffold to be successfully, it must have open and interconnected pores that are essential for cellular nutrition, migration, proliferation, vascularization and formation of new tissues [4]; pore density between 60 and 95\%, pore sizes approximately $100 \mu \mathrm{m}$ which improves cell-scaffold interaction and pore sizes between $300 \mu \mathrm{m}-500 \mu \mathrm{m}$ which improves vascularization [5]. There are several techniques used for the manufacture of 3D scaffolds, such as salt leaching, gas forming, phase separation and freeze - drying, the disadvantage of those is that they do not allow to control with precision the internal architecture of the scaffolds, while with the technology 3D printing is feasible because it uses a computer program design to define certain parameters of porosity and architecture of the piece you want to obtain [6]. For this reason we believe that it is an advantage to obtain a biomaterial in a filament format that is compatible with 3D printing technology.

The objective of this project was to develop a printable biomaterial by $3 \mathrm{D}$ technology (filament), composed of PLA and HA, to be used as 3D printing raw material and thus generate 
custom scaffolds for trabecular bone regeneration. In addition, these scaffolds will be subjected to an irradiation process in order to be sterilized and as a mechanism to control their rate of degradation to be compatible with bone regeneration.

\section{MATERIALS AND METHODS}

\subsection{PLA / HA filament fabrication}

The manufacture of PLA filaments loaded with HA was a process that was executed in three steps. The first consisted of the dissolution of high molecular weight PLA (NatureWorks) pellets with different concentrations of commercial HA in an organic solution, which was eliminated from the final product by a heating process. In the next step the PLA / HA composite was pulverized by the use of an electric grinder. Finally, the mixture was incorporated into the hopper of a Thermo Scientific mini-processor (Process 11) to obtain the desired filament.

Three types of PLA filaments were extruded by adding HA concentrations of $10 \%, 5 \%$ and $3 \%$ w / w. These composites with the same mass were characterized by Differential Scanning Calorimetry (DSC) in order to determine changes in the thermal properties of the material, Infrared Spectroscopy by Fourier Transform (FTIR) to evaluate the presence of functional groups characteristic of the materials used and the absence of the solvent using the homogenization of it.

\subsection{Printing and radiation of scaffold prototypes}

The filaments were used to make the impressions by the additive method in a machine. Then the printed pieces of $20 \mathrm{~mm} \times 20 \mathrm{~mm}$ x $1 \mathrm{~mm}$ were irradiated with doses of 15 and $25 \mathrm{kGy}$.

\subsection{Biological tests performed on scaffold prototypes}

The prototypes were tested for cytotoxicity (MTT test) according to ISO 10993 part 5 to check the safety of them and cell adhesion tests to qualitatively determine the affinity of the cells for the material, this was evaluated using SEM images. Both tests were evaluated using a Vero cell line. 


\subsection{Hydrolytic degradation assays}

A preliminary test of accelerated hydrolytic degradation was carried out on the printed and irradiated prototypes, in order to predict how the material is degraded in the long term. It was used as a guide for the development of the same, ASTM F 1635, ISO 10993-13 and ISO 13781 standards.

The samples were subjected to a high temperature in order to simulate the long-term degradation in short times. A temperature $10^{\circ} \mathrm{C}$ lower than the Glass transition temperature $(\mathrm{Tg})$ of the material was chosen. The filament with $3 \% \mathrm{HA}$ has a $\mathrm{Tg}$ of $53.3^{\circ} \mathrm{C}$, therefore the test was carried out at $43^{\circ} \mathrm{C}$.

To quantify the process of degradation of the pieces, the weight of the pieces was measured at different times. The samples were analyzed in periods of time that were calculated considering the aging of the material from one week to 19.5 months.

The control of this test was carried out with PLA pieces without subjecting them to gamma radiation.

\section{RESULTS}

\subsection{PLA / HA filament fabrication}

The diameter of the obtained filaments was evaluated, this data is important since there are two suitable sizes of filaments for use in 3D printers, one of them is $1.75 \mathrm{~mm}$ and the other is $2.8 \mathrm{~mm}$. The values of diameters obtained (Table 1) of the three types of extruded filaments showed that the three filaments (Figure 1) are apt to be used in a typical 3D printer as they approach the values of commercial filaments. 
Table 1: Diameter of the obtained filaments

\begin{tabular}{cc}
\hline \% of HA & Filament diameters $(\mathbf{m m})$ \\
\hline 3 & $1.61 \pm 0.25$ \\
5 & $1.52 \pm 0.27$ \\
10 & $1.34 \pm 0.09$ \\
\hline
\end{tabular}

HA: Hydroxyapatites

Figure 1: Filaments. On the left PLA/3\% HA $w / w$, in the middle PLA/5\% HA $w / w$ and on the right PLA/10\% HA w/w.

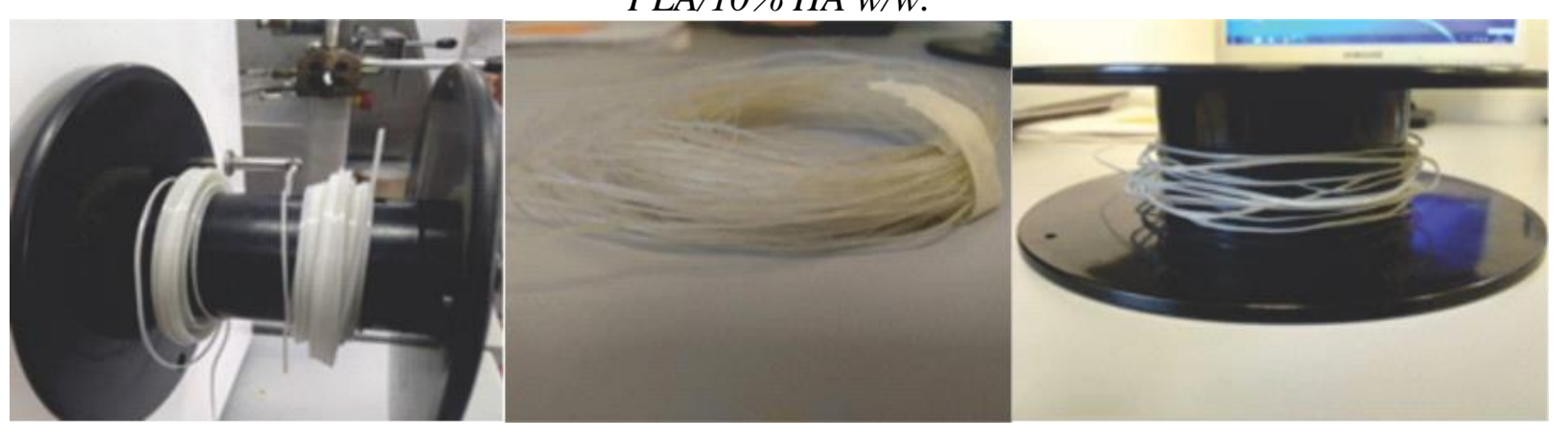

\subsection{DSC and FTIR Tests}

The three filaments were subjected to DSC and FTIR tests. In Figure 2 the DSC curve of the three filament types are shown simultaneously. In red the PLA / 3\% HA is observed, in blue PLA / 5\% HA and in pink the DSC curve of the PLA / 10\% HA filament is displayed. In the image, the Tg is also shown with a circle on the left and the melting temperature (Tf) with the circle on the right. 
Figure 2: Superposition of three DSC curve corresponding to: red (PLA/3\% HA), blue (PLA/5\% HA) and pink PLA/10\% HA).

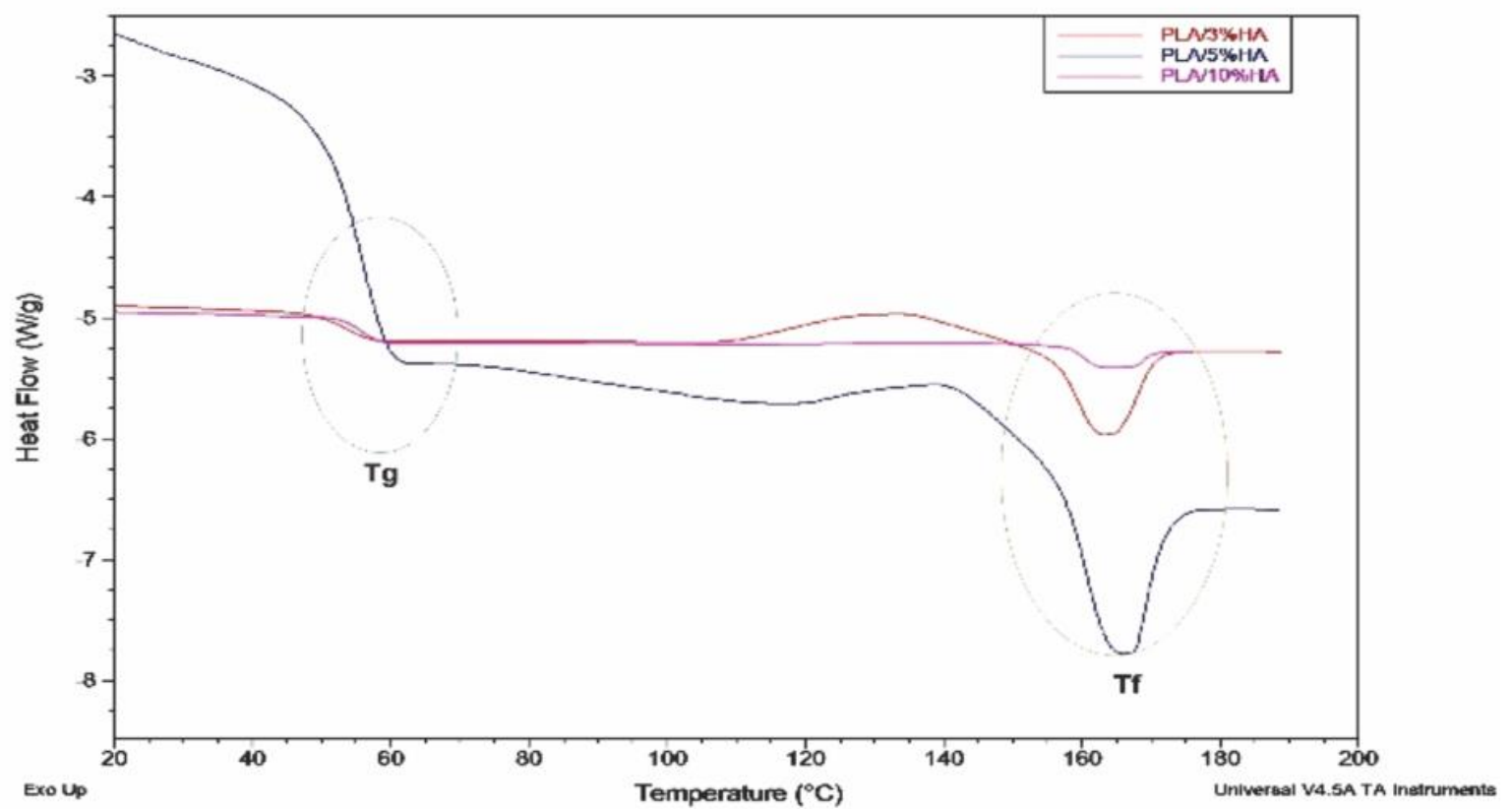

Table 2 shows the relevant data obtained from these DSC curve

Table 2: $\mathrm{Tg}$ and $\mathrm{Tf}$ data for each filament.

\begin{tabular}{ccc}
\hline Type of filament & $\mathbf{T g}\left({ }^{\circ} \mathbf{C}\right)$ & Tf $\left({ }^{\circ} \mathbf{C}\right)$ \\
\hline PLA/3\% HA & 53.68 & 163.14 \\
PLA/5\% HA & 58.11 & 165.17 \\
PLA/10\% HA & 55.91 & 163.46 \\
\hline
\end{tabular}

The DSC curve is essential to know the phase transitions that occur in the material when it is subjected to temperature variations. The temperature values of $\mathrm{Tg}$ and $\mathrm{Tf}$ presented in table 2 indicate that they did not vary with the different HA loads, which makes the extrusion and printing process stable and reproducible. That is, for the three types of filaments, the same extrusion profile and the same temperature can be used in the printing nozzle. Fig. 3 shows the normalized FTIR spectra of the filaments with the different HA loads. 
Figure 3: FTIR spectra of the filaments: in red PLA/3\% HA, in blue (PLA/5\% HA) and in blackPLA/10\% HA).

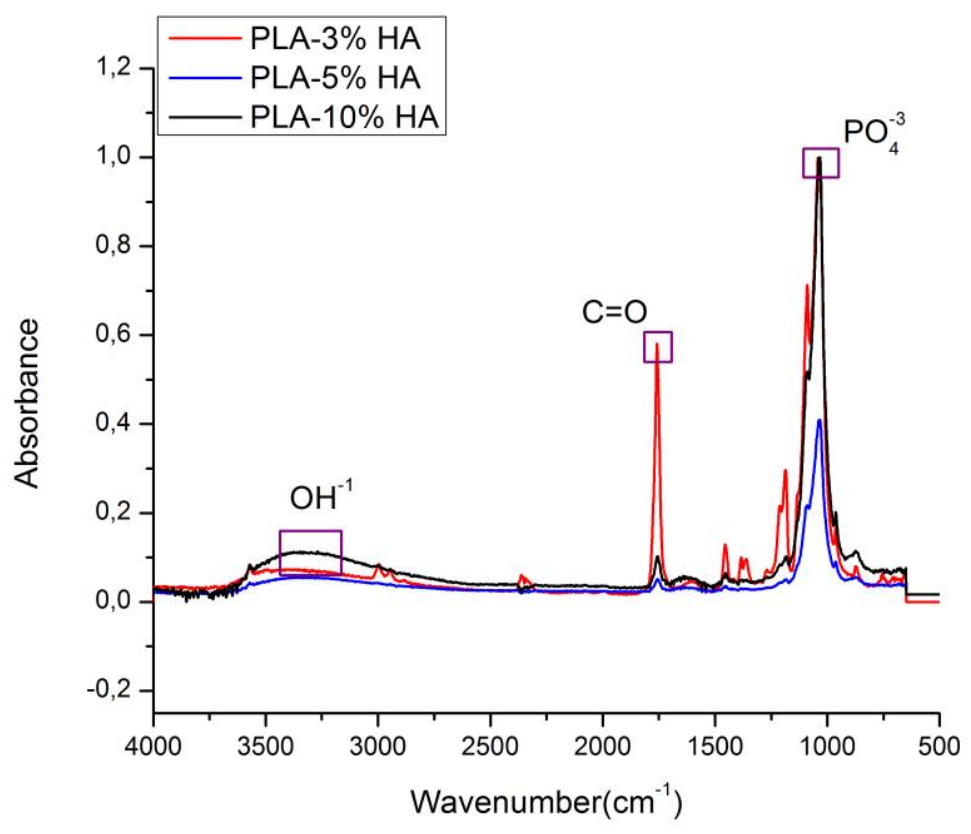

These FTIR spectra show an intense band at $1030 \mathrm{~cm}^{-1}$ that form part of the phosphate groups of the $\mathrm{HA}$, another band at $1750 \mathrm{~cm}^{-1}$ that indicates the presence of the $\mathrm{C}=\mathrm{O}$ group of the PLA. And finally a peak of very low intensity is displayed in the region of $3500-3000 \mathrm{~cm}^{-1}$ corresponding to the $\mathrm{OH}$ groups present in the PLA and can also be found in HA [6], in this region, it is only observed that there is an increase in the intensity of the peak corresponding to PLA-10\% HA, which may be due to the higher HA content.

The analysis of the spectators allows us to corroborate the organic solvent used for the mixture of the PLA with the HA and to identify the functional groups both the PLA and the HA which is important since they must appear in the spectra.

In addition, in the area of low frequencies, the peak of the phosphate group presents greater intensity than those corresponding to the PLA, generating a shielding of the same. Therefore, in this spectrum only one peak appears in the place of the three characteristics of the PLA.

A measure that reduces the concentration of $\mathrm{HA}$ in the composite material, the characteristic peaks of the PLA appear with greater intensity, however, it does not have the peak of the phosphate group of the HA. 


\subsection{Printing and radiation of scaffolding prototypes}

Because the PLA filament with 3\% improved HA printing, it has been designed to make the first three-dimensional prints. In a 3D printing machine, prototypes were manufactured (Figure 4), and used to perform cytotoxicity, cell adhesion and degradation tests.

Figure 4: $3 D$ Printed prototypes of PLA/3 \%HA composite


\subsection{Biological tests performed on scaffolding prototypes}

The cytotoxicity assays shown cell viability greater than $70 \%$, indicating that they are not cytotoxic. Table 3 shows the results of this assays. On average, for $15 \mathrm{kGy}$ the cell viability was $94.3 \% \pm 5.39 \%$ while for $25 \mathrm{kGy}$ the cell viability was $99.03 \% \pm 6.90 \%$.

Table 3: Cytotoxic assay.

\section{Sample / Extraction medium Absorbed dose (kGy) \% Cell viability}

$\begin{array}{ccc}\text { PLA/3\% HA/MEM } & 15 & 92.57 \\ \text { PLA/3\%HA/MEM } & 15 & 89.52 \\ \text { PLA/3\% HA/PBS } & 15 & 100.00 \\ \text { PLA/3\% HA/MEM } & 25 & 91.70 \\ \text { PLA/3\% HA/MEM } & 25 & 105.40 \\ \text { PLA/3\% HA/PBS } & 25 & 100.00\end{array}$

MEM: Minimum essential medium, PBS: Phosphate buffered saline 
The results of the adhesion test are exhibited by the following SEM images of Figure 5. The negative control, prototype without cell seeding, is observed in Figure 5 a) and the prototype that is included in the cells is presented in Figure $5 \mathrm{~b}$ ), here the outline of the cell is marked with yellow to highlight its morphology.

Figure 5: SEM images of adhesion test. a) Control sample. b) Sample with cells.

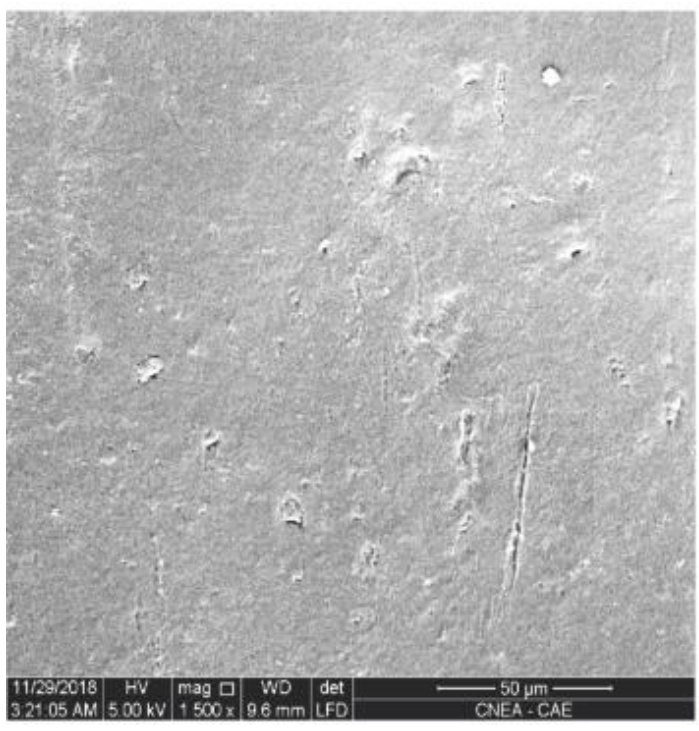

a)

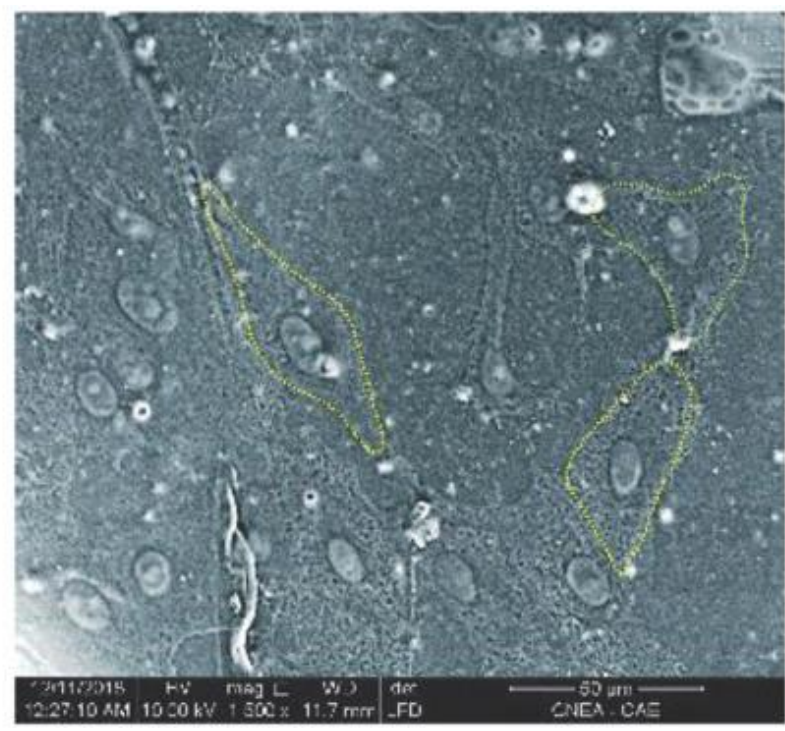

b)

It can be seen in figure $5 \mathrm{~b}$ ) that a large number of cells are displayed and they appear stretched with their central nuclei and forming agglomerations. This is contrasted with the image of the control sample in which no cellular content is observed.

\subsection{Hydrolytic degradation study}

Accelerated hydrolytic degradation tests at 19.5 months showed that the specimen irradiated at $15 \mathrm{kGy}$ showed a degradation of $0.16 \%$ and the specimen irradiated at $25 \mathrm{kGy}$ showed a degradation of $0.13 \%$. The control sample under the same conditions showed no degradation. 


\section{CONCLUSION}

Firstly, from the characterization of the PLA filaments with different HA loads, similar data were obtained that were not conclusive to facilitate the choice of the filament, however, we decided to choose the filament with charge of $3 \% \mathrm{HA}$ since it showed greater stability during printing without causing problems in the process of it, it proved to be more tenacious than the rest of the elaborated filaments and besides having a diameter similar to the commercial one it can be used as raw material of any printer $3 \mathrm{D}$ without too many requirements.

Once the most appropriate percentage of HA was chosen, the prototypes were printed and then sterilized by radiation with doses of $15 \mathrm{kGy}$ or $25 \mathrm{kGy}$. The cytotoxicity tests showed that both types of prototypes were non-cytotoxic and allowed cell adhesion. The degradation test showed that both prototypes subjected to radiation had degradation unlike the control sample that did not show changes during the in vitro test. However, the irradiated prototypes had a very slow hydrolytic degradation rate compared to expect. We believe that this is due to the incorporation of HA that generates nucleation centers that may be reducing the rate of degradation. For this reason, we are evaluating testing higher doses of radiation since even though the radiation rate was low, we consider that by increasing the dose we will be able to better control the degradability of the material. We also intend to carry out in vivo degradation tests, since the enzymatic action of other proteins and cellular activity are involved in this medium.

So far the results are encouraging since we were able to obtain a printable biomaterial, noncytotoxic and optimal for cell adhesion, therefore we can say that our filament would seem to be suitable for the printing of customized 3D scaffolds to be used as bone replacement to avoid the morbidity of the patient when having to extract bone from a healthy area.

\section{REFERENCES}

[1] AMINI, A. R.; LAURENCIN, C. T.; NUKAVARAPU, S. P. Bone tissue engineering: recent advances and challenges. Critical Reviews ${ }^{\text {TM }}$ in Biomedical Engineering, 40(5) (2012). 
[2] FISHER, J. P.; REDDI, A. H. Functional tissue engineering of bone: signals and scaffolds. Topics in tissue engineering, 1, 1-29 (2003).

[3] DURAN, H. A.; BALleSTEROS, D. Y.; CORREO, N. M. Adhesión de osteoblastos sobre andamios de PLA-PLG-biocerámico-colágeno, fotosensibilizados con luz UV. Revista ION, 27(2) (2014).

[4] LOH, Q. L.; CHOONG, C. Three-dimensional scaffolds for tissue engineering applications: role of porosity and pore size. Tissue Engineering Part B: Reviews, 19(6), 485-502 (2013).

[5] ANNABI, N.; NICHOL, J. W.; ZHONG, X., J; KOSHY, S.; KHADEMHOSSEINI, A,; DEHGHANI, F. Controlling the porosity and microarchitecture of hydrogels for tissue engineering. Tissue Engineering Part B: Reviews, 16(4), 371-383 (2010).

[6] RÍOS MENESES, T. Fabricación de material compuesto de pla reforzado con cerámico bioactivo para la fabricación de material de osteosíntesis. Tesis de Ingeniería de Materiales. Universidad de Antioquia .Medellín (2016). 